\title{
Wheat and Hazelnut Inspection with Impact Acoustics Time-Frequency Patterns
}

Nuri F. Ince,

Department of Electrical and Computer Engineering, University of Minnesota USA

Ibrahim Onaran ,

Department of Electrical and Electronics Engineering, Bilkent University, Turkey

Ahmed H. Tewfik,

Department of Electrical and Computer Engineering, University of Minnesota USA

Habil Kalkan,

Informatics Institute, Middle East Technical University, Turkey

Tom Pearson,

United States Department of Agriculture - Agricultural Research Service, USA

A. Enis Cetin,

Department of Electrical and Electronics Engineering, Bilkent University, Turkey

Yasemin Yardimci,

Informatics Institute, Middle East Technical University, Turkey

\author{
Written for presentation at the \\ 2007 ASABE Annual International Meeting \\ Sponsored by ASABE \\ Minneapolis Convention Center \\ Minneapolis, Minnesota \\ 17 - 20 June 2007
}

\begin{abstract}
Kernel damage caused by insects and fungi is one of the most common reason for poor flour quality. Cracked hazelnut shells are prone to infection by cancer producing mold. We propose a new adaptive time-frequency classification procedure for detecting cracked hazelnut shells and damaged wheat kernels using impact acoustic emissions recorded by dropping wheat kernels or


hazelnut shells on a steel plate. The proposed algorithm is based on a flexible local discriminant bases (F-LDB) procedure. The F-LDB method combines local cosine packet analysis and a frequency axis clustering approach which supports individual time and frequency band adaptation. Discriminant features are extracted from the adaptively segmented acoustic signal, sorted according to a Fisher class separability criterion, post processed by principal component analysis and fed to linear discriminant. We describe experimental results that establish the superior performance of the proposed approach when compared with prior techniques reported in the literature or used in the field. Our approach achieved classification accuracy in paired separation of undamaged wheat kernels from IDK, Pupae and Scab damaged kernels with 96\%, 82\% and 94\%. For hazelnuts the accuracy was $97.1 \%$.

Keywords. Acoustic measurement, time-frequency analysis, adaptive signal processing, pattern classification.

The authors are solely responsible for the content of this technical presentation. The technical presentation does not necessarily reflect the official position of the American Society of Agricultural and Biological Engineers (ASABE), and its printing and distribution does not constitute an endorsement of views which may be expressed. Technical presentations are not subject to the formal peer review process by ASABE editorial committees; therefore, they are not to be presented as refereed publications. Citation of this work should state that it is from an ASABE meeting paper. EXAMPLE: Author's Last Name, Initials. 2007. Title of Presentation. ASABE Paper No. 07xxxx. St. Joseph, Mich.: ASABE. For information about securing permission to reprint or reproduce a technical presentation, please contact ASABE at rutter@asabe.org or 269-429-0300 (2950 Niles Road, St. Joseph, MI 49085-9659 USA). 


\section{Introduction}

In this paper we address two important food inspection problems using a novel adaptive time frequency segmentation technique: detection of cracked hazelnut and wheat kernel damage. Damaged or cracked hazelnut shells are more prone to aflatoxin producing molds (Aspergillus Flavus), which can cause cancer. Therefore it is crucial to remove hazelnuts with damaged shells from a given shipment prior to processing.

Wheat kernel damage caused by insects and fungi are the main sources of flour quality degradation. Fusarium graminearum, a fungi found in wheat, creates "scab" damage and may lead to toxins known to cause cancer (Christensen et al., 1986). Furthermore internal insect infestation degrades the quality and value of wheat and is one of the most difficult defects to detect. This type of kernel damage occurs when an adult female insect chews a small hole into the kernel, deposits its egg, and then seals the egg with a mixture of mucus. In the "pupae" stage, the egg plug is the same color as the wheat surface so it is nearly impossible to detect by visual inspection. When the egg hatches, the insect larvae develops and exits the kernel by chewing an exit tunnel and forming what is called an "Insect Damaged Kernel" (IDK). This type of kernel damage causes grain loss by consumption, nutritional losses, and degradation in the end-use quality of flour (Christensen et al., 1986). Separation of the damaged wheat kernels from the undamaged ones is crucial for health and quality. Therefore their percentage in the production/market is limited by USDA and industry set standards (USDA. Electronic code of federal regulations).

Several methods have been studied to tackle the problem of separating damaged wheat kernels from good ones, including X-Ray imaging and carbon dioxide measurements. However these methods are slow and expensive. Recently impact acoustic emission has been successfully used to separate IDK kernels from healthy ones (Pearson et al., 2005). This idea was borrowed from a successful sorting system developed by some of the authors for separating open and close pistachio shells. The system is based on dropping the pistachio shells from a certain height on a metal plate and processing the resulting impact acoustics for discrimination (Cetin et al., 2004). Speech processing algorithms including the combination of time domain modeling, spectral features and short time variances were used widely (Pearson et al., 2005) for classification. The system exhibited high accuracy when separating IDK from healthy kernels. However, the authors reported poor results on other types of kernel damage, such as scab and pupae infested kernels. The algorithms used in (Cetin et al., 2004) were also recently extended to separate cracked hazelnuts shells from undamaged ones. Specifically, the authors of (Kalkan et al. 2006) report classification accuracy around 90\% in cracked and healthy hazelnut shell separation.

Here we investigate a new cracked hazelnut and wheat kernel damage detection method based on an adaptive time-frequency (t-f) analysis of the impact acoustic signals generated by wheat kernels or hazelnut shells when dropped from a fixed height. The proposed approach requires no prior knowledge on the relevant time and frequency indexes of impact acoustics signals. It implements an arbitrary time and frequency tiling with flexible local discriminant bases algorithm. This algorithm is obtained by combining local cosine packet analysis and a frequency axis clustering approach that supports individual time and frequency band adaptation. The adaptively extracted t-f features are then processed by principal component analysis and classified with linear discriminant analysis.

This paper is organized as follows. In the next section we describe the data acquisition system to record impact acoustic emissions. Then we explain the adaptive time-frequency segmentation algorithm. In section IV we proceed with the principal component analysis to reduce the dimensionality of feature set and finally use a linear discriminant for classification. In 
section 4 we present experimental results on wheat and hazelnuts data sets to show the superior performance of the introduced approach. A schematic diagram describing the overall classification system is shown in Figure1.

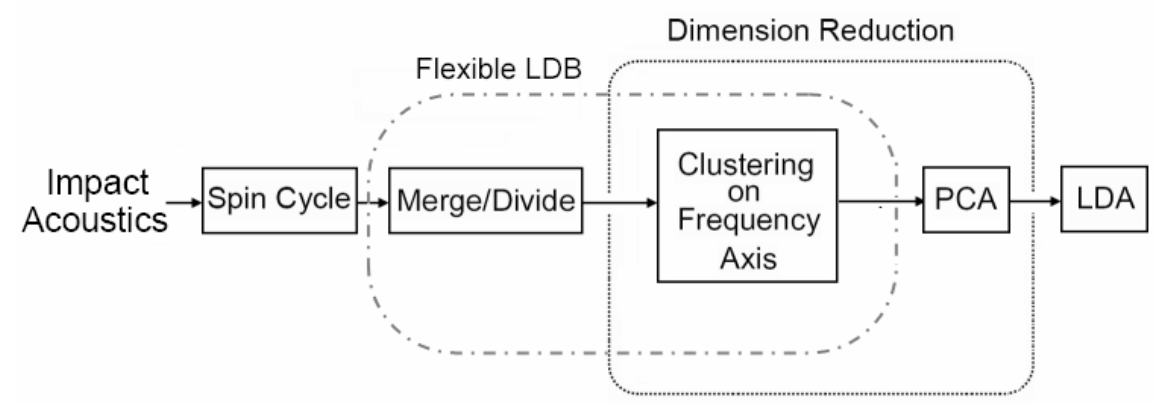

Figure 1. The block diagram of the proposed signal processing and classification system

\section{DATA ACQUISITION}

A schematic of the experimental apparatus for singulating wheat kernels and hazelnuts, dropping them onto the impact plate, then collecting the acoustic emissions from the impact is shown in Figure 2. The impact plate is a polished block of stainless steel approximately $7.5 \mathrm{x}$ $5.0 \times 10 \mathrm{~cm}$. The mass of the impact plate is much larger than that of the wheat kernels in order to minimize vibrations from the plate interfering with acoustic emissions from kernels. A microphone, which is sensitive to frequencies up to $100 \mathrm{KHz}$, is used in order to sense especially ultrasonic acoustic emissions from the wheat kernels. Microphone signals were digitized at a sampling frequency of $192 \mathrm{KHz}$ and $44.1 \mathrm{kHz}$ with 16 bit resolution for wheat and hazelnuts respectively. The data acquisition was triggered using an optical sensor. Sample signals recorded with the data acquisition system are given in Figure 3.

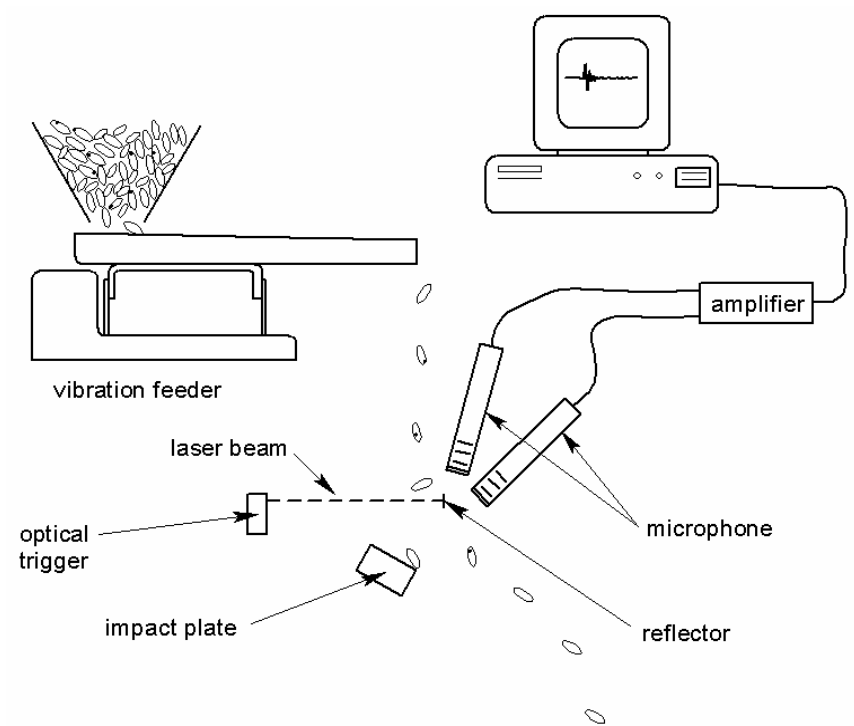

Figure 2. Schematic of wheat sorter based on acoustic emissions. Modified from (Pearson et al., 2005). 

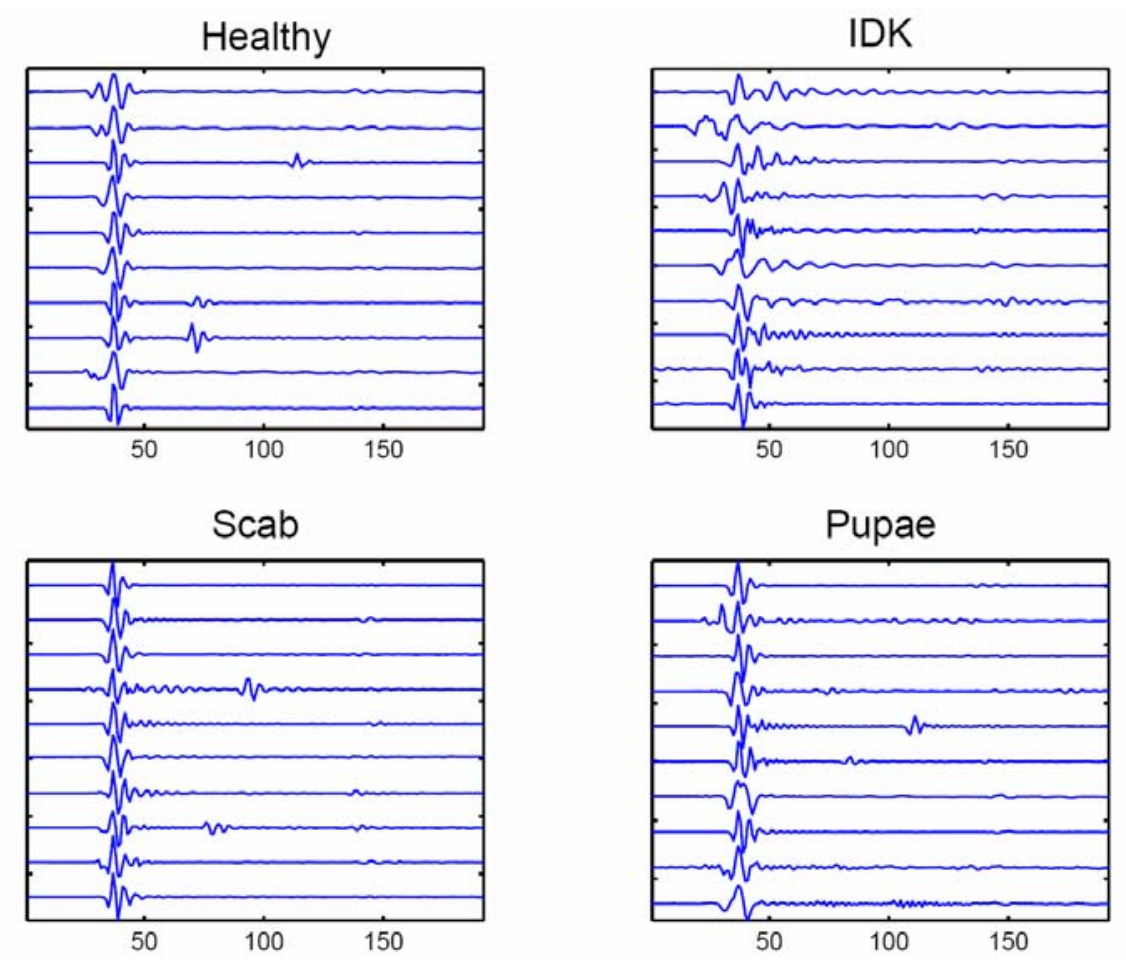

(a)

Healthy
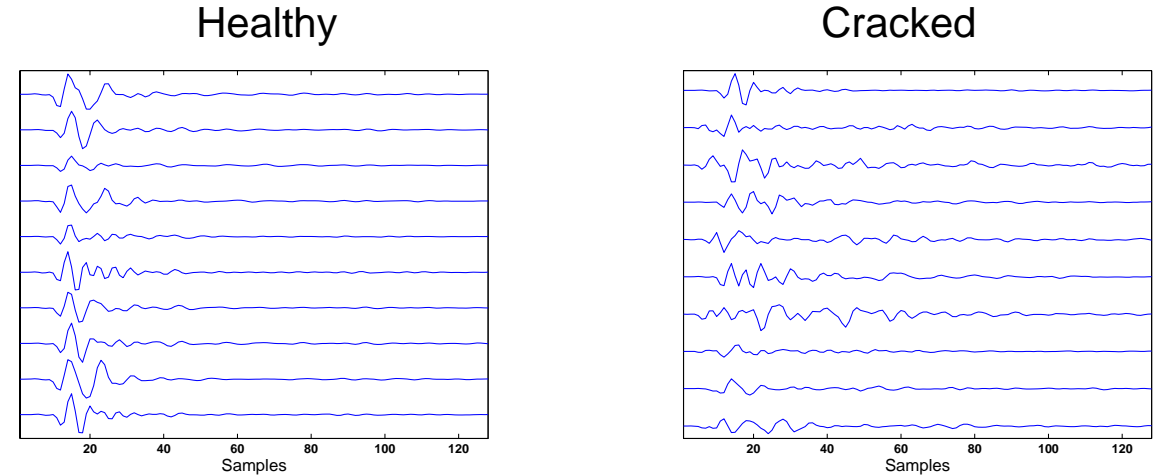

(b)

Figure 3. Sample Waveforms: (a) Wheat Kernel Impact acoustics for 4 types. (b) Undamaged and Cracked hazelnut records. $(\mathrm{N}=10)$

\section{SIGNAL PROCESSING}

We begin by describing the Flexible-Local Discriminant Bases algorithm (F-LDB) of (Ince et al., 2006). As mentioned earlier, we will use this procedure to adaptively segment the timefrequency plane and extract relevant features for classification. We detail the post processing of time frequency plane features in section IV.

\section{Construction Adaptive Time Frequency Segmentation}

Transient features in a signal can carry significant information. Mostly such features are omitted due to their low energy or improper analysis that ignores temporal information. In order to extract such information it is important to focus on local properties of the signal. The F-LDB was 


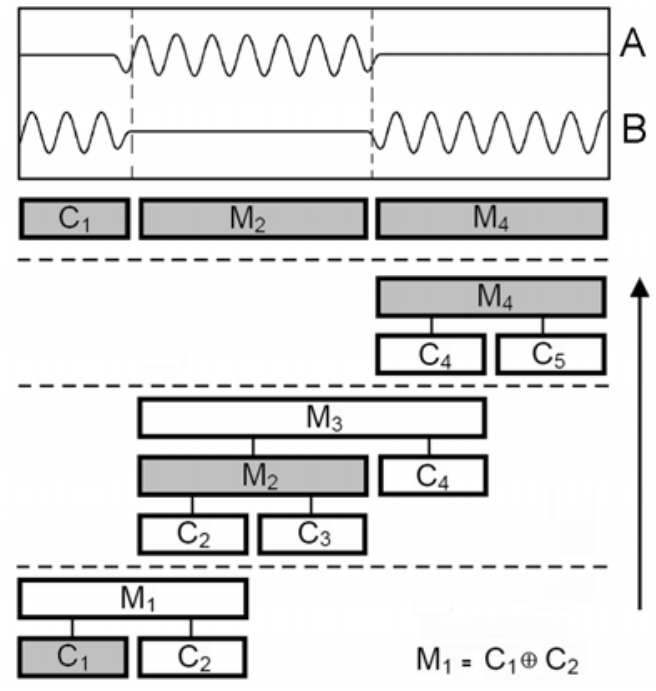

(a)

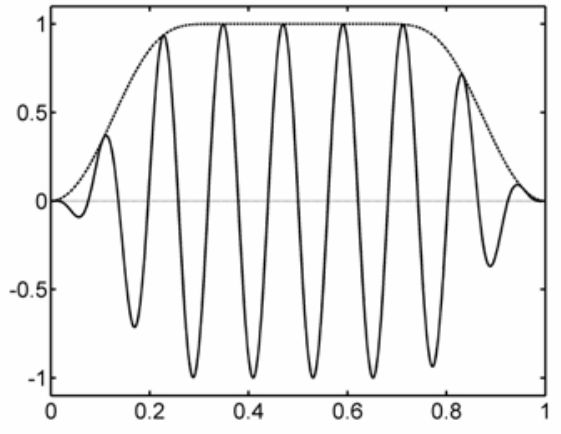

(b)

Fig 4. A schematic illustration of merge/divide based segmentation. It finds the segments where classes A and $\mathbf{B}$ are well separated. (b) The smooth cosine basis used to represent the signal in given segments of (a).

developed to extract such local information by constructing adaptive time-frequency tilings for discrimination (Ince et al., 2006). The F-LDB algorithm is an enhanced version of original LDB which is limited dyadic/pyramidal tree structure (Saito et al., 2002). The F-LDB algorithm expands the signal into orthonormal bases by using local trigonometric transform in consecutive time segments and finds those segments where the distance between classes are maximized. Since the Cosine Packets are not shift invariant, we first apply the spin cycle procedure of (Ince at al., 2006 and Saito et al., 2002) to the training and test data as indicated in Figure 1. The reader is referred to (Saito et al., 2002) for further detail

Let us explain how the adaptive time segmentation procedure works on sample signals given in Figure 4 in a two class problem. The signals $A$ and $B$ are analyzed in adjacent windows with a children and mother structure as indicated from bottom to top. The windows are smooth and overlap neighboring windows (See Figure 4. (b)). In each window, the signals are expanded using Local Cosine Packets. Then the distance between the cumulative distributions functions corresponding to the resulting expansion coefficients are compared in the mother and children subspaces. Whenever the total distance between the classes in the children subspaces is larger than that in the mother subspace, the signal is divided at that point. Otherwise, the children segments are merged. In the next iteration, the merged segment is used as the left child. Note that the right child is the smallest size time segment used by the procedure and will have length less than that of the left segment. Unlike the dyadic case, the children windows are not necessarily half the length of the mother window as illustrated in Figure 4. This algorithm is iterated along the time axis by implementing the above procedure on adjacent segments. The resulting merge operations can cause the mother window and left child to grow to be multiples of the basic smallest segment or time partitioning can occur after each comparison.

The cumulative probability distributions of the expansion coefficients corresponding to the two classes are estimated via high resolution histogram derived from the training data set. 


\section{Dimension Reduction and Classification}

The F-LDB algorithm produces a feature space of high dimensionality equal to the dimension of the original signal. In order reduce the dimensionality and deal with the uncertainty in frequency axis we adaptively group the expansion coefficients in each adapted time segment. We reshape the Merge/Divide strategy for frequency axis. Here consecutive frequency indices were merged only if their union has larger discrimination power than that of the individual indices treated separately. The procedure described above is basically a clustering approach by cost function maximization and produces adaptive frequency segmentation suitable for discrimination in each time segment.

Once the t-f segmentation is completed we use the Fisher class separability criterion

$$
F=\frac{\left(\mu_{1}-\mu_{2}\right)^{2}}{\sigma_{1}^{2}+\sigma_{2}^{2}}
$$

for sorting the features, where and are the mean and standard deviation of the feature they correspond to.

As a last step, principal component analysis (PCA) was implemented on the top discriminant feature set. We projected the top subset of the sorted feature set onto its eigenvectors.

$$
u_{k}=W_{k}^{T} x
$$

where is the projected vector, is the kth eigenvector of the covariance matrix of the feature set and is the feature vector. The PCA processed top feature set was input to a linear discriminant analysis (LDA). The weight vector used in LDA is calculated as

$$
v=\left(\sum_{1}+\sum_{2}\right)^{-1}\left(m_{1}-m_{2}\right)
$$

where, are the covariance matrix and mean of class features respectively. The distance of a feature vector to the discriminating hyperplane is calculated as

$$
d=v^{T} x
$$

where is the feature vector and is the distance.

\section{RESULTS}

In this section we present experimental results on the classification of wheat kernels and hazelnuts. The impact acoustic signals corresponding to wheat kernels (Healthy, IDK, Scab and Pupae) were 1024 samples long while those corresponding to cracked and healthy hazelnut shells were 960 samples long. The minimum window size used for the time segmentation with the flexible LDB procedure size was selected to be 16 samples for wheat and 32 samples for hazelnuts. The smooth overlapping part of the windows was set to half of the minimum window size. A one spin cycle procedure is used before processing the signals. After completing the t-f segmentation, the features were converted to log scale, normalized and sorted by the $F$ criterion. The top 128 features were processed by PCA and sorted according the eigenvalues in descending order. A 2 times 2 fold cross validation was implemented to estimate classification error. In order to compare the efficiency of the introduced algorithm we used the same features of (Cetin et al., 2004) and refer as base approach (BA). The reader is referred to (Cetin et al., 2004) for further detail. 
Table 1 shows results from both approaches obtained on wheat kernels. IDK, SCAB, PUPAE and undamaged (UD) indicate the individual classification error for each type.

TABLE 1. The classification accuracies obtained with Flexible-LDB (F-LDB) and base approaches (BA) in separating undamaged kernels (UD) from damages ones. The results belong to paired classifications.

\begin{tabular}{llll}
\hline & UD - IDK & UD - SCAB & UD - PUPAE \\
\hline F-LDB & $96 \%-95.7 \%$ & $93.9 \%-96.9 \%$ & $84 \%-81.2 \%$ \\
BA & $92 \%-82.3 \%$ & $68.4 \%-66.3 \%$ & $69.8 \%-66.5 \%$ \\
\hline
\end{tabular}

The proposed approach outperformed the conventional methods, especially for the scab and pupae damaged kernels. We visualize the t-f locations selected by F-LDB method in Figure 5 . Interestingly, the impact area and several locations after impact onset were selected by the algorithm. These features appear to be the critical ones and resulted in a lower error rate and we believe they might be related to the vibration of metal plate. We also tested the classification performance by constructing single time-frequency segmentation for discriminating the healthy kernels from all undamaged types. When such a strategy is used the pupae classification accuracy rate drops to $62 \%$. The t-f maps partly explain the degradation in performance. As seen from Figure 5. (a) and (c) the t-f location selected for pupae and scab are different.

In order to preserve the high classification accuracy for all types we propose a step wise discrimination strategy. In case a new signal is recorded it will be iteratively classified by all setups. However a high true positive rate is necessary for such a system. In the current system the most critical classifier is the one for Pupae kernels. We plot the Receiver Operator Characteristic Curve in Figure 6 for this classifier output. The current work point is shown with solid arrow. In order to improve the true positive rate to $95 \%$ one will pay with high false positive rate (dashed arrow).

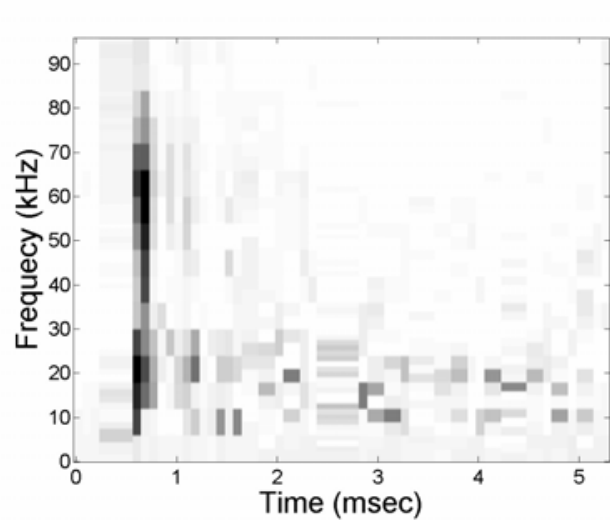

(a)

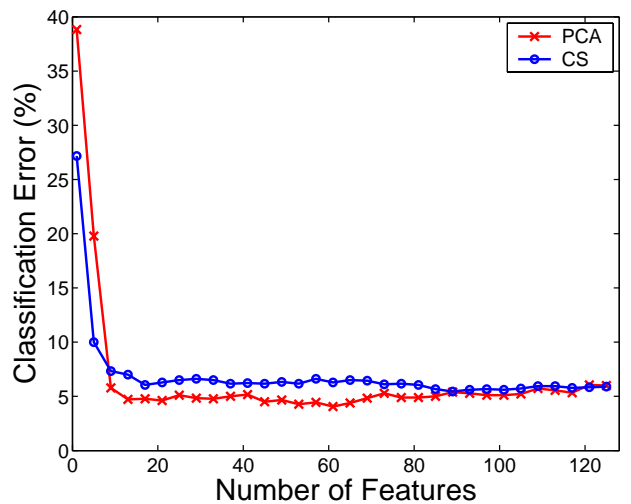

(b) 


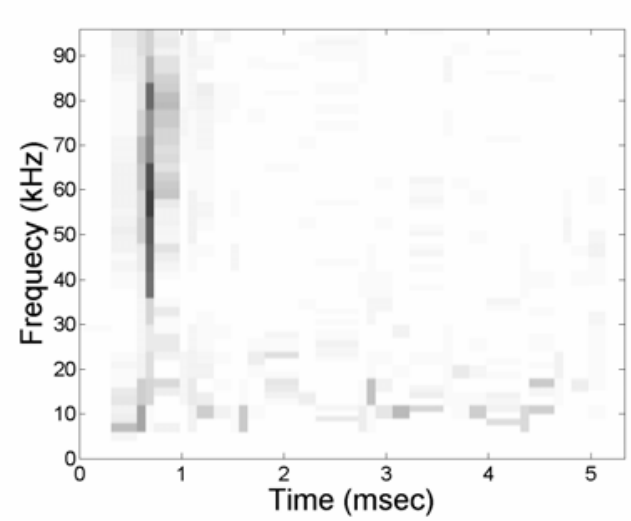

(c)

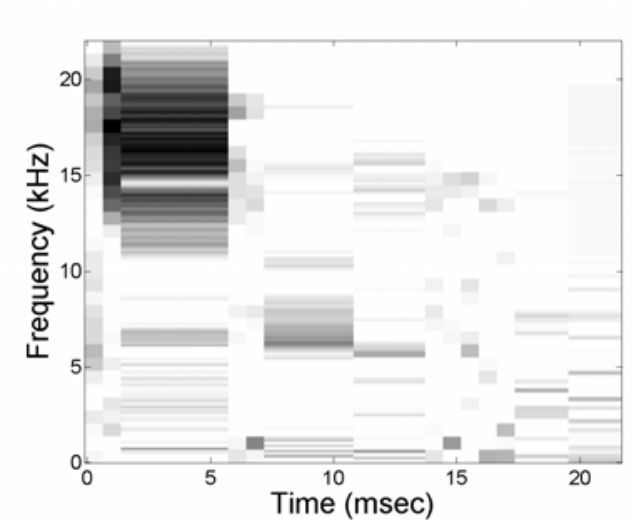

(e)

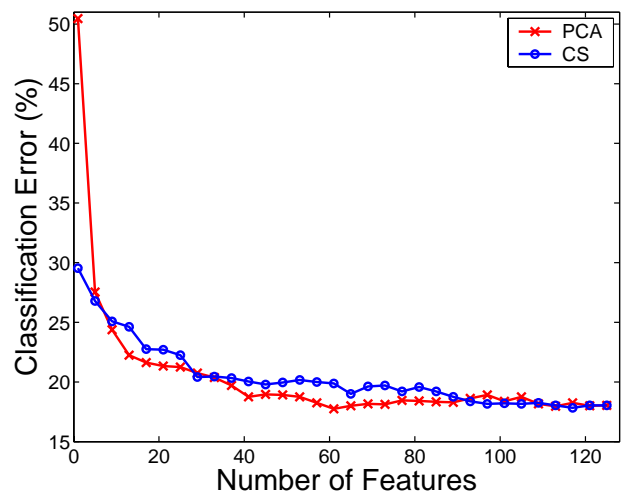

(d)

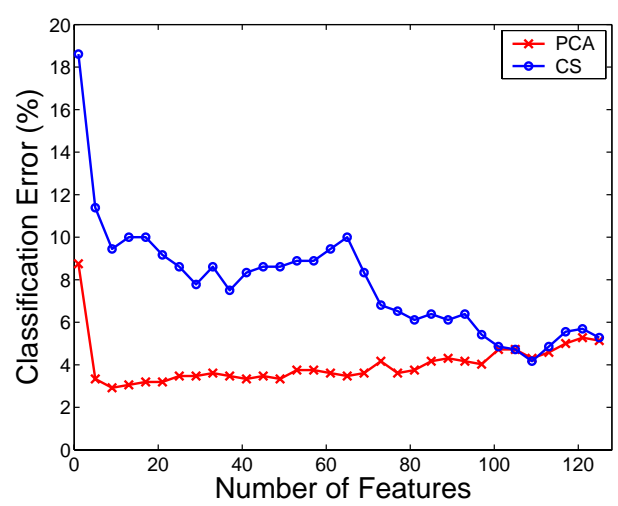

(f)

Figure 5. The left column shows t-f features selected for Scab (a), Pupae (c) and hazelnut (e) classification. The darker features have the more discrimination power. The right column shows the classification error versus the number of features which were sorted by a class separability criteria (CS=F) and PCA. The error curves belong to Scab (b), Pupae (d) and hazelnuts (f) respectively. Notice the lower error rates of PCA with minimal features.

The proposed system has achieved $97.1 \%$ classification accuracy while separating the healthy hazelnut shells from cracked ones. We visualize the discriminant t-f features selected by algorithm in Figure 5.e. The t-f map shows that the most discriminant features are located at high frequency bands following the impact. In Figure 5.f. we see the classification error curve versus the number of features. Here the PCA has improved the classification accuracy from $96 \%$ to $97 \%$ with small number of features. This shows the importance of post processing the t-f features sorted by $\mathrm{F}$ criterion.

As a result, the capacity of adaptively locating the discriminant components and adjusting the t-f segmentations for discrimination makes the proposed algorithm a good candidate for future damaged food kernel separation systems. 


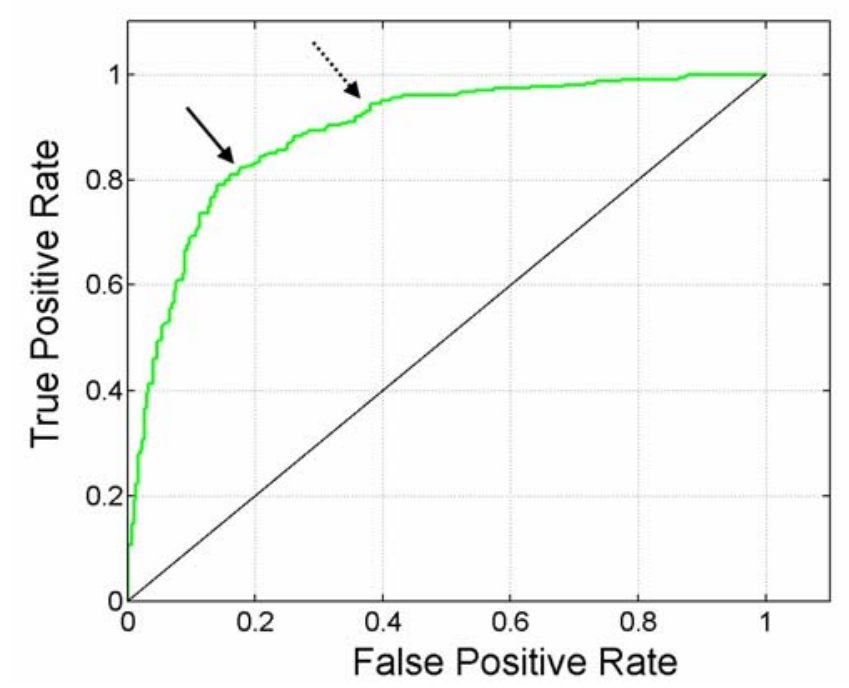

Fig.6. The ROC curve of UD-Pupae classifier output.

\section{References}

Christensen, C.M. and R.A. Meronuck. 1986. "Quality Maintenance in Stored Grains and Seeds." University of Minnesota Press, Minneapolis, MN.

USDA. Electronic code of federal regulations. Title 7 (Agriculture), Chapter VIII (Federal Grain Inspection Service), Part 810 (Official United States Standards for Grain), Available at: www.usda.gov/gispa/lawsandregs/lawsregs.htm,

Pearson, Tom, A. Enis Cetin, Ahmed H. Tewfik and Ron P Haff. 2005. "Feasibility of impactacoustic emissions for detection of damaged wheat kernels",. Digital Signal Processing Elsevier.

Cetin, A.E. ,T.C. Pearson and A. H. Tewfik. 2004. "Classification of closed- and open-shell pistachio nuts using impact acoustical analysis", ICASSP.

Kalkan H. and Y. Yardimci. 2006. "Classification of hazelnuts by impact acoustics". IEEE MLSP.

INCE, N. Firat, Sami ARICA and Ahmed TEWFIK. 2006. "Classification of Single Trial Motor Imagery EEG recordings by using Subject Adapted Non-Dyadic Arbitrary TimeFrequency Tilings " J. Neural Eng. 3 235-244

Saito N., R.R. Coifman, F. B. Geshwind and F. Warner, 2002. "Discriminant feature extraction using empirical probability density and a local basis library", Pattern Recognition, vol.35, 1842-1852. 